\title{
A class of uncertain variational inequality problems
}

\author{
Qiqiong Chen and Yuanguo Zhu*
}

${ }^{*}$ Correspondence:

ygzhu@njust.edu.cn

Department of Applied

Mathematics, Nanjing University of

Science and Technology, Nanjing,

Jiangsu 210094, People's Republic

of China

\begin{abstract}
In this paper, we propose a new class of variational inequality problems, say, uncertain variational inequality problems based on uncertainty theory in finite Euclidean spaces $R^{n}$. It can be viewed as another extension of classical variational inequality problems besides stochastic variational inequality problems. Note that both stochastic variational inequality problems and uncertain variational inequality problems involve uncertainty in the real world, thus they have no conceptual solutions. Hence, in order to solve uncertain variational inequality problems, we introduce the expected value of uncertain variables (vector). Then we convert it into a classical deterministic variational inequality problem, which can be solved by many algorithms that are developed on the basis of gap functions. Thus the core of this paper is to discuss under what conditions we can convert the expected value model of uncertain variational inequality problems into deterministic variational inequality problems. Finally, as an application, we present an example in a noncooperation game from economics.
\end{abstract}

Keywords: variational inequality; uncertainty theory; optimization; descent method; line search

\section{Introduction}

The variational inequality problem (VIP for short) is an important discipline of mathematics. Over the past several decades, VIP has developed into a very fruitful discipline in the field of mathematical programming. The developments include a rich mathematical theory, a host of effective solution algorithms, a multitude of interesting connections to numerous disciplines such as optimization theory, economics, engineering, game theory and networks (see [1-8]) and so forth. Although there are many derivatives of VIP in infinite spaces such as Hilbert spaces, Banach spaces etc. from the classical VIP of $R^{n}$, we just pay our attention to the finite dimensional Euclidean space $R^{n}$.

Consider the problem of finding a point $x \in S \subset R^{n}$ such that

$$
(y-x)^{T} F(x) \geq 0, \quad \forall y \in S,
$$

where $S$ is a nonempty closed convex subset of $R^{n}$, and $F: S \rightarrow R^{n}$ is a mapping. If there exists an element $x^{*}$ satisfying (1) for every $y \in S$, then $x^{*}$ is called a solution to the variational inequality (1). Then the problem to find a solution to variational inequality (1) is called a variational inequality problem associated with the mapping $F$ and the subset $S$,

(c) 2015 Chen and Zhu. This article is distributed under the terms of the Creative Commons Attribution 4.0 International License (http://creativecommons.org/licenses/by/4.0/), which permits unrestricted use, distribution, and reproduction in any medium, provided you give appropriate credit to the original author(s) and the source, provide a link to the Creative Commons license, and indicate if changes were made. 
which is denoted by $\operatorname{VIP}(F, S)$. The set of solutions to this problem is denoted by $\operatorname{Sol}(S, F)$. Note that what the underlying mapping $F$ in (1) reflects is deterministic, i.e., it does not involve uncertainties. However, there are many instances in practice that the underlying problem not only involves deterministic data, but also contains some uncertain factors in those data. For example, during the transmission of one signal, say sound signal from the base station through relays to the user, it may be interfered by other signals. Hence, in order to reflect those uncertainties, many researchers begin to set foot to the stochastic variational inequality problem (SVIP for short).

Finding a vector $x \in S \subset R^{n}$ such that

$$
P\left\{\theta \in \Theta \mid(y-x)^{T} F(x, \omega(\theta)) \geq 0, \forall y \in S\right\}=1,
$$

where $P$ is the given probability measure of the random variate $\omega$. Or

$$
(y-x)^{T} F(x, \omega(\theta)) \geq 0, \quad \forall y \in S, \theta \in \Theta \text { a.s. }
$$

where $F: S \times \Theta \rightarrow R^{n}$ is a mapping, $\Theta$ is the underlying sample space and a.s. is the abbreviation for 'almost surely' under the given probability measure. Note that problem (3) does not have solutions in general because of the randomness in the underlying function $F$. Therefore, it is natural to give a reasonable reformulation for problem (3). By introducing expected value of $F(x, \omega(\theta))$ over $\omega(\theta)$, we can rewrite problem (3) as follows.

Find a vector $x \in S \subset R^{n}$ such that

$$
(y-x)^{T} E[F(x, \omega(\theta))] \geq 0, \quad \forall y \in S
$$

where $E[F(x, \omega(\theta))]$ is the expected value of $F(x, \omega(\theta))$ over $\omega(\theta)$.

SVIP (4) was investigated in references such as [9-15], etc. And its applications can be found in inventory or pricing competition among several firms that provide substitutable goods or services (see [16] and [17]), a supply chain network model (see [18]), stochastic user equilibrium traffic network (see [19]) and wireless network (see [20]).

On the other hand, in the real world, there are some circumstances when no samples are available in the determinacy phenomena, for example, data of a new stock, data of devastating military experiments, etc., in which it is impossible for us to estimate a probability distribution. It seems that to invite some relevant experts to evaluate their belief degree that each event will happen makes sense. Here the belief degree represents the degree with which we believe the indeterminacy quantity falls into the left side of a given point about the indeterminacy phenomena. There also exist the cases that people's viewpoints may vary from person to person for the same event; even for the same person, his/her view to the event may vary from time to time. Hence, in order to deal with those uncertainty phenomena, Prof. Liu, who came from Tsinghua University of China, founded a new theory - uncertainty theory - in 2007 (see [21]), which is quite different from probability theory. While SVIP is based on probability theory, we present in this paper its counterpart $U V I P$ on the basis of uncertainty theory. There is no doubt that both of them are natural extensions of deterministic VIP.

For solving the deterministic VIP, we can reform it as a system of (unconstraint) equations. We can also cast it as a minimization problem by introducing gap functions [1]. 
Hence, on the basis of gap functions, we can design a host of effective solution algorithms for solving VIP.

In 1992, Fukushima [22] designed a regularized gap function as $g(x)=\max \left\{F(x)^{T}(x-\right.$ $\left.y)-\frac{1}{2}\|x-y\|^{2}\right\}$. Then, on the basis of it, he casted a VIP as a constrained differentiable optimization problem in the sense that any global solution of the reformulated optimization problem is a solution to VIP, and vice versa. It is presented as the following theorem.

(Theorem 3.3 in [22]) Assume that the mapping $F: R^{n} \rightarrow R^{n}$ is continuously differentiable and its Jacobian matrix $\nabla F(x)$ is positive definite for all $x \in S$. If $x$ is a stationary point of problem $\min _{x \in S} g(x)$, i.e.,

$$
\langle\nabla g(x), y-x\rangle \geq 0 \quad \text { for all } y \in S
$$

then $x$ is a global optimal solution of problem $\min _{x \in S} g(x)$, and hence it solves the variational inequality (1).

His research shows that the objective function is continuously differentiable whenever the mapping involved in the VIP is continuously differentiable (see Theorem 3.2 in [22]). Moreover, he discussed descent methods for solving the equivalent problem (see Lemma 5 in Section 2).

We highlight that the main idea for this paper is to discuss how to convert a UVIP into a classical VIP. Then one can solve it by many mature algorithms, which can be documented in $[1,22]$.

This paper is organized as follows. In Section 2, we review some definitions and lemmas which are useful in the sequel; in Section 3, we present a class of uncertain variational inequalities, discuss its expected value model and convert it into a class of classical deterministic variational inequalities in detail; as an application, we investigate in Section 4 an example developed from economics; we conclude the paper with Section 5.

\section{Preliminaries}

Before starting our discussion, in this section, let us review a few necessary definitions and lemmas.

We first state the axiomatic system of uncertainty theory as follows.

Let $\Gamma$ be a nonempty set (universal set), and let $\mathcal{L}$ be a $\sigma$-algebra over $\Gamma$. Then $(\Gamma, \mathcal{L})$ is called a measurable space and each element $\Lambda$ in $\mathcal{L}$ is called a measurable set, which is also called an event in uncertainty theory.

Definition 1 (Liu [21]) A set function $\mathcal{M}$ defined on the $\sigma$-algebra $\mathcal{L}$ over $\Gamma$ is called an uncertain measure if it satisfies the following three axioms.

Axiom 1 (Normality axiom) $\mathcal{M}(\Gamma)=1$ for the universal set $\Gamma$.

Axiom 2 (Duality axiom) $\mathcal{M}\{\Lambda\}+\mathcal{M}\left\{\Lambda^{c}\right\}=1$ for any event $\Lambda$.

Axiom 3 (Subadditivity axiom) For every countable sequence of events $\Lambda_{1}, \Lambda_{2}, \ldots$, we have

$$
\mathcal{M}\left\{\bigcup_{i=1}^{\infty} \Lambda_{i}\right\} \leq \sum_{i=1}^{\infty} \mathcal{M}\left\{\Lambda_{i}\right\} .
$$


Remark 1 Uncertain measure is interpreted as the personal belief degree (not frequency) of an uncertain event that may occur. It depends on the personal knowledge concerning the event. The uncertain measure will change if the state of knowledge changes.

Similar to stochastic variable defined on a probability space, an uncertain variable is a real-valued function defined on an uncertainty space. A formal definition is given as follows.

Definition 2 (Liu [21]) An uncertain variable is a function $\xi$ from an uncertainty space $(\Gamma, \mathcal{L}, \mathcal{M})$ to the set of real numbers such that $\{\xi \in \mathcal{B}\}$ is an event for any Borel set $B$. A $k$-dimensional uncertain vector is a function $\xi$ from an uncertainty space $(\Gamma, \mathcal{L}, \mathcal{M})$ to the set of $k$-dimensional real vectors such that $\xi \in B$ is an event for any Borel set $B$ of $k$-dimensional real vector.

Note that the vector $\xi=\left(\xi_{1}, \xi_{2}, \ldots, \xi_{k}\right)$ is an uncertain vector if and only if $\xi_{1}, \xi_{2}, \ldots, \xi_{k}$ are uncertain variables.

In order to describe uncertain variables, Liu [21] introduced a new concept: uncertainty distribution. Note that it is a carrier of incomplete information of uncertain variable.

Definition 3 (Liu [21]) The uncertainty distribution $\Phi$ of an uncertain variable $\xi$ is defined by

$$
\Phi(x)=\mathcal{M}\{\xi \leq x\}
$$

for any real numbers.

As a special uncertainty distribution, a linear uncertainty distribution which is denoted by $\mathcal{L}(a, b)$ is

$$
\Phi(x)= \begin{cases}0, & \text { if } x \leq a ; \\ \frac{x-a}{b-a}, & \text { if } a \leq x \leq b ; \\ 1, & \text { if } x \geq b,\end{cases}
$$

where $a$ and $b$ are real numbers with $a<b$.

Besides the uncertainty distribution, we present the regular uncertainty distribution as follows, which is used more often.

Definition 4 (Liu [23]) An uncertainty distribution $\Phi(x)$ is said to be regular if it is a continuous and strictly increasing function, with respect to $x$ at which $0<\Phi(x)<1$, and

$$
\lim _{x \rightarrow-\infty} \Phi(x)=0, \quad \lim _{x \rightarrow+\infty} \Phi(x)=1
$$

Remark 2 It is clear that a regular uncertainty distribution $\Phi(x)$ has an inverse function on the range of $x$ with $0<\Phi(x)<1$, and the inverse function $\Phi^{-1}(\alpha)$ exists on the open interval $(0,1)$. Hence we have the following definition of inverse uncertain distribution. 
Definition 5 (Liu [23]) Let $\xi$ be an uncertain variable with regular uncertainty distribution $\Phi(x)$. Then the inverse function $\Phi^{-1}(\alpha)$ is called the inverse uncertainty distribution of $\xi$.

Since the expected value is the average value of the uncertain variable in the sense of uncertain measure and it represents the size of uncertain variable, it is natural to present the expected value of an uncertain variable as follows.

Definition 6 (Liu [21]) Let $\xi$ be an uncertain variable. Then the expected value of $\xi$ is defined by

$$
E(\xi)=\int_{0}^{+\infty} \mathcal{M}\{\xi \geq x\} \mathrm{d} x-\int_{-\infty}^{0} \mathcal{M}\{\xi \leq x\} \mathrm{d} x
$$

provided that at least one of the two integrals is finite.

It is easy to know that if an uncertain variable $\xi \sim \mathcal{L}(a, b)$, i.e., it is subject to a linear uncertainty distribution, then it has an expected value $E[\xi]=\frac{a+b}{2}$.

Since the independence between uncertain variables is very important while describing many results, we state it formally.

Definition 7 (Liu [24]) The uncertain variables $\xi_{1}, \xi_{2}, \ldots, \xi_{n}$ are said to be independent if

$$
\mathcal{M}\left\{\bigcap_{i=1}^{n}\left(\xi_{i} \in B_{i}\right)\right\}=\bigwedge_{i=1}^{n} \mathcal{M}\left\{\xi_{i} \in B_{i}\right\}
$$

for any Borel sets $B_{1}, B_{2}, \ldots, B_{n}$.

Definition 8 (Liu [21]) Suppose that $\xi, \xi_{1}, \xi_{2}, \ldots$ are uncertain variables defined on the uncertainty space $(\Gamma, \mathcal{L}, \mathcal{M})$. The sequence $\left\{\xi_{i}\right\}$ is said to converge almost surely to $\xi$ if there exists an event $\Lambda$ with $\mathcal{M}(\Lambda)=1$ such that

$$
\lim _{i \rightarrow \infty}\left|\xi_{i}(\gamma)-\xi(\gamma)\right|=0
$$

for every $\gamma \in \Lambda$. In that case we write $\xi_{i} \rightarrow \xi$, a.s.

Next, we present some lemmas which will be used in the discussion that follows.

Lemma 1 (Liu [21]) Let $\xi$ be an uncertain variable with uncertainty distribution $\Phi(x)$. Then

$$
E(\xi)=\int_{0}^{\infty}(1-\Phi(x)) \mathrm{d} x-\int_{-\infty}^{0} \Phi(x) \mathrm{d} x
$$

Lemma 2 (Liu [23]) Let $\xi$ be an uncertain variable with regular uncertainty distribution $\Phi(x)$. Then

$$
E(\xi)=\int_{0}^{1} \Phi^{-1}(\alpha) \mathrm{d} \alpha .
$$


Lemma 3 (Liu and $\mathrm{Ha}[25])$ Assume $\xi_{1}, \xi_{2}, \ldots, \xi_{n}$ are independent uncertain variables with regular uncertainty distributions $\Phi_{1}, \Phi_{2}, \ldots, \Phi_{n}$, respectively. If $f\left(x_{1}, x_{2}, \ldots, x_{n}\right)$ is strictly increasing with respect to $x_{1}, \ldots, x_{m}$ and strictly decreasing with respect to $x_{m+1}, \ldots, x_{n}$, then the uncertain variable $\xi=f\left(\xi_{1}, \xi_{2}, \ldots, \xi_{n}\right)$ has an expected value

$$
E(\xi)=\int_{0}^{1} f\left(\Phi_{1}^{-1}(\alpha), \ldots, \Phi_{m}^{-1}(\alpha), \Phi_{m+1}^{-1}(1-\alpha), \ldots, \Phi_{n}^{-1}(1-\alpha)\right) \mathrm{d} \alpha
$$

where $\Phi_{i}^{-1}(i=1,2, \ldots, n)$ are the inverse uncertainty distributions of $\xi_{i}(i=1,2, \ldots, n)$.

Note that a constant $x$ is also an uncertain variable with which its inverse distribution is $x$ itself. If we take $\xi_{1}=\mathbf{x} \in R^{n}$ in Lemma 3, then we have the next lemma naturally.

Lemma 4 Assume that $f\left(\mathbf{x}, \xi_{1}, \ldots, \xi_{n}\right)$ is strictly increasing with respect to $\xi_{1}, \ldots, \xi_{m}$ and strictly decreasing with respect to $\xi_{m+1}, \ldots, \xi_{n}$. If $\xi_{1}, \ldots, \xi_{n}$ are independent uncertain variables with uncertainty distributions $\Phi_{1}, \ldots, \Phi_{n}$, respectively, then the expected function $E\left[f\left(\mathbf{x}, \xi_{1}, \ldots, \xi_{n}\right)\right]$ is equal to

$$
\int_{0}^{1} f\left(\mathbf{x}, \Phi_{1}^{-1}(\alpha), \ldots, \Phi_{m}^{-1}(\alpha), \Phi_{m+1}^{-1}(1-\alpha), \ldots, \Phi_{n}^{-1}(1-\alpha)\right) \mathrm{d} \alpha
$$

where $\Phi_{i}^{-1}(i=1,2, \ldots, n)$ are the inverse uncertainty distributions of $\xi_{i}(i=1,2, \ldots, n)$.

Since a VIP can be converted into an equivalent optimization problem through a regularized gap function (refer to Theorem 3.1 and Theorem 3.3 in [22]). Here we give the definition of a regularized gap function. Note that this terminology was not used by Fukushima in [22], but the function he defined was really a gap function by his later work [26].

Definition 9 (Facchinei and Pang [1]) Let the $\operatorname{VIP}(F, S)$ be given with $F$ defined on an open set $\Omega$ containing $S$. Let $\alpha$ be a positive constant and let $G$ be a symmetric positive definite matrix. The regularized gap function of $\operatorname{VIP}(F, S)$ is defined as

$$
g_{\alpha}=\sup _{y \in S}\left\{F(x)^{T}(x-y)-\frac{\alpha}{2}\|x-y\|_{G}^{2}\right\}
$$

for all $x$ in $\Omega$, where $\|x\|_{G}^{2}=\sqrt{x^{T} G x}$ is the $G$-norm of $x \in R^{n}$. We note immediately that $g_{\alpha} \geq g_{\beta}, \forall x \in \Omega$ for any two scalars $\alpha>\beta>0$.

Facchinei and Pang discussed many properties of $g_{\alpha}$; for more details, please refer to the book [1].

Definition 10 (Facchinei and Pang [1]) Let $S$ be a convex subset of $R^{n}$ and $G \in R^{n \times n}$ be a symmetric positive definite matrix. The projection operator $\operatorname{Proj}_{S, G}: R^{n} \rightarrow S$ is called the skewed projection mapping onto $S$ if for every fixed $x \in R^{n}, \operatorname{Proj}_{S, G}(x)$ is the solution of the following convex optimization problem:

$$
\left\{\begin{array}{l}
\min \frac{1}{2}\|y-x\|_{S}^{2}=\frac{1}{2}(y-x)^{T} S(y-x) \\
\text { s.t. } y \in S
\end{array}\right.
$$


Lemma 5 (Proposition 4.1 in [22]) Let the mapping $F: R^{n} \rightarrow R^{n}$ be continuously differentiable. If the Jacobian matrix $\nabla F(x)$ is positive definite on $S$, then for each $x \in S$ the vector $d=H(x)-x$ satisfies the descent condition

$$
\langle\nabla g(x), d\rangle<0, \quad \text { whenever } d \neq 0,
$$

where $g(x)=F(x)^{T}(x-H(x))-\frac{1}{2}\|x-H(x)\|_{G}^{2}, H(x)=\operatorname{Proj}_{S, G}\left(x-G^{-1} F(x)\right)$.

Following Fukushima [22], we can give the following lemma.

Lemma 6 Let the mapping $F: R^{n} \rightarrow R^{n}$ be continuously differentiable. If the Jacobian matrix $\nabla F(x)$ is positive definite on $S$, then for each $x \in S$ the vector $d_{\alpha}=H_{\alpha}(x)-x$ satisfies the descent direction

$$
\left\langle\nabla g_{\alpha}(x), d_{\alpha}\right\rangle<0
$$

whenever $d_{\alpha} \neq 0$, where $g_{\alpha}(x)$ is as defined in Definition 9 and $H_{\alpha}(x)=\operatorname{Proj}_{S, G}(x-$ $\left.\alpha^{-1} G^{-1} F(x)\right)$.

Proof Similar to the proof of Proposition 4.1 in [22] just replace the mapping $H$ with $H_{\alpha}$.

From Lemma 6, we can execute the descent methods to find a solution to the VIP related; for more details, one can refer to [22].

\section{Main results}

We are now in the position to reveal the main results of this paper. Invoked by the definition of stochastic variational inequality problems, we present the definition of uncertain variational inequality problems first.

Consider the uncertain variational inequality problems (UVIP for short): finding a vector $x \in S \subset R^{n}$ such that

$$
\mathcal{M}\left\{\gamma \in \Gamma \mid(y-x)^{T} F(x, \xi(\gamma)) \geq 0, \forall y \in S\right\}=1,
$$

where $\mathcal{M}$ is the given uncertain measure of the uncertain variable $\xi$. Or

$$
(y-x)^{T} F(x, \xi(\gamma)) \geq 0, \quad \forall y \in S, \forall \gamma \in \Lambda \text { a.s., }
$$

where $S$ is a closed convex subset of $R^{n}, \xi(\gamma)$ is an uncertain variable defined on an uncertainty space $(\Gamma, \mathcal{L}, \mathcal{M}), \Lambda$ is an event with $\mathcal{M}(\Lambda)=1, F: R^{n} \times R \rightarrow R^{n}$ is a mapping, and a.s. is the abbreviation for 'almost surely' under the given uncertain measure.

Since there is no solution to problem (6), by introducing the expected value of $F(x, \xi(\gamma))$ over $\xi(\gamma)$, we rewrite (6) as finding a vector $x \in S$ such that

$$
(y-x)^{T} E[F(x, \xi(\gamma))] \geq 0, \quad \forall y \in S
$$

Take $f(x)=E[F(x, \xi(\gamma))]$, then $U V I P(7)$ can be written as

$$
(y-x)^{T} f(x) \geq 0, \quad \forall y \in S .
$$


Note that if $f(x)$ can be evaluated either analytically or numerically, then (8) can be regarded as a deterministic VIP and consequently can be solved by many mature numerical methods, which can be documented in $[1,22]$.

Hence we now investigate under what circumstances $f(x)$ can be evaluated in uncertainty theory.

Theorem 1 Let $\xi$ be an uncertain variable with regular uncertainty distribution $\Phi(t)$. Let $F(\mathbf{x}, y)$ be a function defined on $R^{n} \times R$. If $F(\mathbf{x}, y)$ is strictly increasing with respect to $y$, $\nabla_{\mathbf{x}} F(\mathbf{x}, y)$ and $F(\mathbf{x}, y)$ are continuous on $R^{n} \times R$, then

$$
f(\mathbf{x})=E[F(\mathbf{x}, \xi)]=\int_{0}^{1} F\left(\mathbf{x}, \Phi^{-1}(\alpha)\right) \mathrm{d} \alpha
$$

is continuous on $R^{n}$ and, moreover,

$$
\nabla_{\mathbf{x}} f(\mathbf{x})=\int_{0}^{1} \nabla_{\mathbf{x}} F\left(\mathbf{x}, \Phi^{-1}(\alpha)\right) \mathrm{d} \alpha
$$

where $\nabla_{\mathbf{x}} F(\mathbf{x}, y)$ is the gradient of $F(\mathbf{x}, y)$ with respect to the vector $\mathbf{x} \in R^{n}$ and $\nabla_{\mathbf{x}} f(\mathbf{x})$ is analogous.

Proof Since $\xi$ is an uncertain variable with regular uncertainty distribution $\Phi(t)$, its inverse uncertainty distribution $\Phi^{-1}(\alpha)$ continuously exists from Definition 4 and Definition 5. Because $F(\mathbf{x}, y)$ is continuous on $R^{n} \times R$, thus $F\left(\mathbf{x}, \Phi^{-1}(\alpha)\right)$ is continuous on $R^{n} \times[0,1]$ by extending the continuity of $\Phi^{-1}(\alpha)$ to the endpoint. Then, from Lemma 4 in conjunction with the continuity property of parametric integral, we know that $f(\mathbf{x})=$ $E[F(\mathbf{x}, \xi)]=\int_{0}^{1} F\left(\mathbf{x}, \Phi^{-1}(\alpha)\right) \mathrm{d} \alpha$ is continuous on $R^{n}$. Furthermore, we arrive at the identity (9) from the analysis of matrix and when $\nabla_{\mathbf{x}} F(\mathbf{x}, y)$ is continuous $R^{n} \times R$, so is $\nabla_{\mathbf{x}} f(\mathbf{x})=$ $\int_{0}^{1} \nabla_{\mathbf{x}} F\left(\mathbf{x}, \Phi^{-1}(\alpha)\right) \mathrm{d} \alpha$ by the differentiable property of parametric integral. This completes the proof.

On the other hand, when $F(\mathbf{x}, y)$ is strictly decreasing, we have the following result.

Corollary 1 Let $\xi$ be an uncertain variable with regular uncertainty distribution $\Phi(t)$. Let $F(\mathbf{x}, y)$ be a function defined on $R^{n} \times R$. If $F(\mathbf{x}, y)$ is strictly decreasing with respect to $y$, $\nabla_{\mathbf{x}} F(\mathbf{x}, y)$ and $F(\mathbf{x}, y)$ are continuous on $R^{n} \times R$, then

$$
f(\mathbf{x})=E[F(\mathbf{x}, \xi)]=\int_{0}^{1} F\left(\mathbf{x}, \Phi^{-1}(1-\alpha)\right) \mathrm{d} \alpha
$$

is continuous on $R^{n}$ and, moreover,

$$
\nabla_{\mathbf{x}} f(\mathbf{x})=\int_{0}^{1} \nabla_{\mathbf{x}} F\left(\mathbf{x}, \Phi^{-1}(1-\alpha)\right) \mathrm{d} \alpha,
$$

where $\nabla_{\mathbf{x}}$ is the gradient operator in terms of $\mathbf{x}$.

Proof From Lemma 4, what we need to do is to replace $\Phi^{-1}(\alpha)$ in the proof of Theorem 1 with $\Phi^{-1}(1-\alpha)$ since the inverse distribution of uncertain variables in these two situations is different. 
As an extension of Theorem 1 and Corollary 1 to uncertain vector, we present the following result.

Theorem 2 Let $\xi=\left(\xi_{1}, \xi_{2}, \ldots, \xi_{k}\right)$ be a $k$-dimensional uncertain vector, $\mathbf{x}$ be a vector in $R^{n}$, $\mathbf{y}=\left(y_{1}, y_{2}, \ldots, y_{k}\right)$ be a vector in $R^{k}$. Let $F(\mathbf{x}, \mathbf{y}): R^{n} \times R^{k} \rightarrow R$ be a function which is strictly increasing with respect to $y_{1}, \ldots, y_{m}$ and strictly decreasing with respect to $y_{m+1}, \ldots, y_{k}$. Assume that $\xi_{i}(i=1,2, \ldots, k)$ are independent uncertain variables with regular uncertainty distributions $\Phi_{i}(t)(i=1,2, \ldots, k)$, respectively. If both $F(\mathbf{x}, \mathbf{y})$ and its gradient $\nabla_{\mathbf{x}} F(\mathbf{x}, \mathbf{y})$ are continuous on $R^{n} \times R^{k}$, then

$$
\begin{aligned}
f(\mathbf{x}) & =E[F(\mathbf{x}, \xi)] \\
& =\int_{0}^{1} F\left(\mathbf{x}, \Phi_{1}^{-1}(\alpha), \ldots, \Phi_{m}^{-1}(\alpha), \Phi_{m+1}^{-1}(1-\alpha), \ldots, \Phi_{k}^{-1}(1-\alpha)\right) \mathrm{d} \alpha
\end{aligned}
$$

is continuous on $R^{n}$ and, moreover,

$$
\nabla_{\mathbf{x}} f(\mathbf{x})=\int_{0}^{1} \nabla_{\mathbf{x}} F\left(\mathbf{x}, \Phi_{1}^{-1}(\alpha), \ldots, \Phi_{m}^{-1}(\alpha), \Phi_{m+1}^{-1}(1-\alpha), \ldots, \Phi_{k}^{-1}(1-\alpha)\right) \mathrm{d} \alpha,
$$

where $\nabla_{\mathbf{x}} f(x)$ is the gradient of $f(x)$ with respect to $\mathbf{x}$.

Proof From Theorem 1 and Corollary 1, in view of Lemma 4, it is easy to derive Theorem 2.

While Theorem 1 and Theorem 2 concern real-valued functions, it is natural for us to extend them to a vector function. Before we start to present the extension, it is necessary to introduce the expected value of an uncertain vector. We give it as follows.

Definition 11 Let $\xi=\left(\xi_{1}, \xi_{2}, \ldots, \xi_{k}\right)$ be a $k$-dimensional uncertain vector. Then we define the expected value of $\xi$ by

$$
E[\boldsymbol{\xi}]=\left(E\left[\xi_{1}\right], E\left[\xi_{2}\right], \ldots, E\left[\xi_{k}\right]\right)
$$

That is, the expected value of an uncertain vector is the vector consisting of the expected value of each component.

Now we are in the position to extend Theorem 2 to vector functions.

Theorem 3 Let $\xi=\left(\xi_{1}, \xi_{2}, \ldots, \xi_{k}\right)$ be a $k$-dimensional uncertain vector whose components are all independent uncertain variables with regular uncertainty distributions $\Phi_{i}(t)$ $(i=1,2, \ldots, k)$. Let $\mathbf{x}$ be a vector in $R^{n}, \mathbf{y}=\left(y_{1}, y_{2}, \ldots, y_{k}\right)$ be a vector in $R^{k}, F(\mathbf{x}, \mathbf{y}): R^{n} \times$ $R^{k} \rightarrow R^{s}$ be a vector-valued function whose components can be expressed as $F(\mathbf{x}, \mathbf{y})=$ $\left(F_{1}(\mathbf{x}, \mathbf{y}), F_{2}(\mathbf{x}, \mathbf{y}), \ldots, F_{s}(\mathbf{x}, \mathbf{y})\right)$. Assume that each $F_{j}\left(\mathbf{x}, y_{1}, \ldots, y_{k}\right)(j=1,2, \ldots, s)$ is strictly increasing with respect to $y_{1}, \ldots, y_{m}$ and strictly decreasing with respect to $y_{m+1}, \ldots, y_{k}$. If both $F_{j}\left(\mathbf{x}, y_{1}, y_{2}, \ldots, y_{k}\right)$ and its Jacobian matrix $\nabla_{x} F_{j}\left(\mathbf{x}, y_{1}, y_{2}, \ldots, y_{k}\right)(j=1,2, \ldots, s)$ are continuous on $R^{n} \times R^{k}$, then

$$
\begin{aligned}
f(\mathbf{x}) & =E[F(\mathbf{x}, \boldsymbol{\xi})]=\left(E\left[F_{1}(\mathbf{x}, \xi)\right], E\left[F_{2}(\mathbf{x}, \boldsymbol{\xi})\right], \ldots, E\left[F_{s}(\mathbf{x}, \xi)\right]\right) \\
& =\left(f_{1}(\mathbf{x}), f_{2}(\mathbf{x}), \ldots, f_{s}(\mathbf{x})\right),
\end{aligned}
$$


where $f_{j}(\mathbf{x})=\int_{0}^{1} F_{j}\left(\mathbf{x}, \Phi_{1}^{-1}(\alpha), \ldots, \Phi_{m}^{-1}(\alpha), \Phi_{m+1}^{-1}(1-\alpha), \ldots, \Phi_{k}^{-1}(1-\alpha)\right) \mathrm{d} \alpha$ is continuous on $R^{n}$ and, moreover,

$$
\nabla_{\mathbf{x}} f(\mathbf{x})=\left(\nabla_{\mathbf{x}} f_{1}(\mathbf{x}), \nabla_{\mathbf{x}} f_{2}(\mathbf{x}), \ldots, \nabla_{x} f_{s}(\mathbf{x})\right)
$$

where $\nabla_{\mathbf{x}} f_{j}(\mathbf{x})=\int_{0}^{1} \nabla_{\mathbf{x}} F_{j}\left(\mathbf{x}, \Phi_{1}^{-1}(\alpha), \ldots, \Phi_{m}^{-1}(\alpha), \Phi_{m+1}^{-1}(1-\alpha), \ldots, \Phi_{k}^{-1}(1-\alpha)\right) \mathrm{d} \alpha(j=1,2, \ldots, s)$ is the gradient of $\nabla_{\mathbf{x}} f_{j}(\mathbf{x})$ with respect to $\mathbf{x}$.

Proof It is easy to derive the results from Theorem 2 in conjunction with Definition 11.

Remark 3 By introducing the expected value of an uncertain variable (vector), from Theorem 3 , after transforming a UVIP into a deterministic VIP, the underlying mapping of the reformulation one is continuous and its Jacobian matrix $\nabla_{\mathbf{x}} f(\mathbf{x})$ can be detected whether positive definite or not. As long as $\nabla_{\mathbf{x}} f(\mathbf{x})$ is positive definite for all $\mathbf{x} \in S$, we can compute the descent direction by Lemma 6 . Then we set $\mathbf{x}^{k+1}=\mathbf{x}^{k}+t^{k} d_{\alpha}^{k}, k=0,1,2, \ldots$, where $d_{\alpha}^{k}$ are given by $d_{\alpha}^{k}=H_{\alpha}\left(\mathbf{x}^{k}\right)-\mathbf{x}^{k}$ and $t^{k} \in[0,1]$ which are determined from $f\left(\mathbf{x}^{k}+t^{k} d_{\alpha}^{k}\right)=$ $\min _{0 \leq t \leq 1} f\left(\mathbf{x}^{k}+t d_{\alpha}^{k}\right)$. Thus we can find a solution to the UVIP by iterations. For more details, please refer to [22]. In fact, Facchinei and Pang [1] presented more algorithms to find a solution to a finite dimensional variational inequality problem on the basis of gap (merit) functions.

\section{An application}

As an application of the preceding results, let us present an example from economics in this section. We develop a supply model of newsvendors who are involved in the production of the same kind of products.

Suppose there are $n$ players in the market who produce the same kind of products. Let $p_{i}$ and $c_{i}$ be the unit price and unit production cost of newsvendor $i$, respectively. Assume that the number of customers who prefer to buy the products from newsvendor $i$ is $D_{i}$, which is an uncertain variable. Customers always purchase the products from their unique preferred newsvendor provided that the products are available. However, a proportion, say $o_{i j}$, of the customers of newsvendor $j$ will purchase the products from newsvendor $i$ if they find that newsvendor $j$ does not have any product left unsold. Let $q_{i}$ denote the nonnegative production level of newsvendor $i$. We group the production level of all newsvendors into the column vector $q \in R_{+}^{n}$. We assume that each newsvendor is faced with a product cost $c_{i} q_{i}$. Give the production level $q_{-i}$ for all other newsvendors. Then, for newsvendor $i$, his revenue is equal to the price he charges for the newspaper times the total quantity which is the minimum of the quantity he produced and the uncertain demand from customers. If we give each newsvendor a limit production level, say $M_{i}$ for newsvendor $i$, we can express the criterion of profit maximization for newsvendor $i$ as

$$
\left\{\begin{array}{l}
\max u_{i}\left(q_{i}, q_{-i}\right) \triangleq p_{i} \min \left(q_{i}, D_{i}+\sum_{j \neq i} o_{i j} \max \left(D_{j}-q_{j}, 0\right)\right)-c_{i} q_{i}, \quad \forall i \\
\text { s.t. } q_{i} \in K_{i} \triangleq\left[0, M_{i}\right] .
\end{array}\right.
$$

Note that $\max \left(D_{j}-q_{j}, 0\right)$ has an economic interpretation which is that for newsvendor $j$, his supply cannot meet the demand of the market. 
Since the profit function $u_{i}\left(q_{i}, q_{-i}\right)$ contains uncertain variables, it cannot be directly maximized. We may maximize its expected value, i.e.,

$$
\left\{\begin{aligned}
\max U_{i}\left(q_{i}, q_{-i}\right)=p_{i} E\left[\min \left(q_{i}, D_{i}+\sum_{j \neq i} o_{i j} \max \left(D_{j}-q_{j}, 0\right)\right)\right]-c_{i} q_{i}, \quad \forall i \\
\text { s.t. } q_{i} \in K_{i} \triangleq\left[0, M_{i}\right] .
\end{aligned}\right.
$$

We assume that all newsvendors compete in a noncooperative fashion, namely play an oligopolistic game by choosing their product level appropriately. Also, we assume that for newsvendor $i$, his/her uncertain demand is $D_{i}=\frac{m}{p_{i}} \xi_{i}$, where $\xi_{1}, \xi_{2}, \ldots, \xi_{n}$ are independent with $E\left[\xi_{i}\right]=e_{i}$ for $i=1,2, \ldots, n$ and $m$ is a constant. It makes sense that when the newsvendor charges more, his/her uncertain demand will decline, and vice versa. Hence, it follows from uncertainty theory that the expected value of the uncertain demand $D_{i}$ is $E\left[D_{i}\right]=\frac{m e_{i}}{p_{i}}$ for all $i$. Then the underlying expected profit function is

$$
U_{i}\left(q_{i}, q_{-i}\right)=p_{i} \min \left(q_{i}, \frac{m e_{i}}{p_{i}}+\sum_{j \neq i} o_{i j} \max \left(\frac{m e_{j}}{p_{j}}-q_{j}, 0\right)\right)-c_{i} q_{i}, \quad \forall i
$$

For convenience, we suppose that for newsvendor $j$, his/her supply cannot meet his/her customers' demand, that is, $D_{j}>q_{j}$, then $\max \left(D_{j}-q_{j}, 0\right)=D_{j}-q_{j}$; furthermore, the expected profit is

$$
U_{i}\left(q_{i}, q_{-i}\right)=p_{i} \min \left(q_{i}, \frac{m e_{i}}{p_{i}}+\sum_{j \neq i} o_{i j}\left(\frac{m e_{j}}{p_{j}}-q_{j}\right)\right)-c_{i} q_{i} .
$$

An optimal solution for the newsvendor's model is a Nash equilibrium. Given that the governing equilibrium, which states that each newsvendor will determine his/her optimal production quantity, given the optimal ones of the competitors, the optimality conditions for all newsvendors simultaneously can be expressed as a variational inequality problem. That is, the equilibrium governing newsvendors' model with competition is equivalent to the solution to the variational inequality problem given as follows: determine $q^{*}=\left(q_{1}^{*}, q_{2}^{*}, \ldots, q_{n}^{*}\right) \in K=\prod_{i=1}^{n} K_{i}$ satisfying

$$
\left(q-q^{*}\right)^{T}\left(-\nabla_{q_{i}} U_{i}\left(q_{i}, q_{-i}\right)\right) \geq 0, \quad \forall q \in K
$$

where $\nabla_{q_{i}}(\cdot, \cdot)$ is the gradient of $U_{i}$ with respect to $q_{i}$ for $i=1,2, \ldots, n$. We now discuss two special cases for the problem.

Case 1: If $q_{i} \geq \frac{m e_{i}}{p_{i}}+\sum_{j \neq i} o_{i j}\left(\frac{m e_{j}}{p_{j}}-q_{j}\right)$, then $U_{i}\left(q_{i}, q_{-i}\right)=m e_{i}-\sum_{j \neq i} o_{i j}\left(\frac{m p_{i} e_{j}}{p_{j}}-p_{i} q_{j}\right)-c_{i} q_{i}$.

Hence $\frac{\partial U_{i}\left(q_{i}, q_{-i}\right)}{\partial q_{i}}=-c_{i}$. Furthermore, for the expected profit maximization problem, it follows from (10) that determine $q^{*} \in K$ satisfying

$$
\left(q-q^{*}\right)^{T} c \geq 0, \quad \forall q \in K
$$

i.e.,

$$
\sum_{i=1}^{n}\left(q_{i}-q_{i}^{*}\right) c_{i} \geq 0, \quad \forall q_{i} \in K_{i}
$$


Case 2: If $q_{i}<\frac{m e_{i}}{p_{i}}+\sum_{j \neq i} o_{i j}\left(\frac{m e_{j}}{p_{j}}-q_{j}\right)$, then $U_{i}\left(q_{i}, q_{-i}\right)=\left(p_{i}-c_{i}\right) q_{i}$.

Hence $\frac{\partial U_{i}\left(q_{i}, q_{-i}\right)}{\partial q_{i}}=p_{i}-c_{i}$. Similarly, we can convert the underlying expected profit maximization problem into the following variational inequality problem: determine $q^{*} \in K$ satisfying

$$
\sum_{i=1}^{n}\left(q_{i}-q_{i}^{*}\right)\left(c_{i}-p_{i}\right) \geq 0, \quad \forall q_{i} \in K_{i}
$$

The optimal condition as expressed in (11) has a nice economic interpretation, which is that a newsvendor will distribute a positive amount of products to the market if the price which the customers are willing to pay is precisely equal to his/her marginal production cost. Note that the production cost function is $c_{i} q_{i}$ and its marginal production cost is $c_{i}$, which is the partial derivative of $c_{i} q_{i}$ with respect to $q_{i}$. If the newsvendor's marginal production cost exceeds what the customers are willing to pay, then he/she will transact none with the demand market.

After finishing the procedure of transformation, we can solve variational inequality problems above by line search algorithm which is stated in Section 3.

Remark 4 We just apply our results in the newsvendor's model. As a matter of fact, there are many problems in practice that can be casted as uncertain variational inequality problems when there exist uncertain variables (vector) such as in electricity supply network [1], user equilibrium [19] and wireless networks [20].

\section{Conclusions}

In this paper, we discussed a new class of variational inequality problems, namely uncertain variational inequality problems based on the uncertainty theory. It can be regarded as another extension of the classical variational inequality problems besides stochastic variational inequality problems. By introducing the expected value model of uncertain variables (vector), we converted one class of uncertain variational inequality problems into a class of deterministic variational inequality problems, which can be solved by many classical methods such as those presented in $[1,22]$. Finally, we applied our results in an economic example.

\section{Competing interests \\ The authors declare that they have no competing interests.}

Authors' contributions

All authors contributed equally and significantly in writing this paper. All authors read and approved the final manuscript.

\footnotetext{
Acknowledgements

The authors gratefully acknowledge the support of the National Natural Science Foundation of China through Grant No. 61273009 and the China Scholarship Council through Grant No. 201406840039. It was accomplished during the first author's visit to Professor Jinlu Li at Shawnee State University, USA. The authors would like to express their deep gratitude for the warm hospitality from Shawnee State University.

Received: 14 April 2015 Accepted: 7 July 2015 Published online: 25 July 2015

References

1. Facchinei, F, Pang, J: Finite-Dimensional Variational Inequalities and Complementarity Problems. Springer, New York (2003)

2. Luo, Z, Pang, J, Ralph, D: Mathematical Programs with Equilibrium Constraints. Cambridge University Press, Cambridge (1996)
} 
3. Yao, J, Guo, J: Variational and generalized variational inequality with discontinuous mappings. J. Math. Anal. Appl. 82(2), 371-392 (1994)

4. Zhang, C: Generalized variational inequalities and generalized quasivariational inequalities. Appl. Math. Mech. 14(4), 315-325 (1993)

5. Zhu, Y: Solutions to variational inequalities of parabolic type. J. Math. Anal. Appl. 321(1), 24-31 (2006)

6. Zhu, Y: Generalized variational inequalities for fuzzy maps. Fuzzy Sets Syst. 69(2), 221-229 (1995)

7. Huang, N, Fang, Y: On vector variational inequalities in reflexive Banach spaces. J. Glob. Optim. 32, 495-505 (2005)

8. $L i, J, Y a o, J:$ The existence of maximum and minimum solutions to general variational inequalities in the Hilbert lattices. Fixed Point Theory Appl. 2011, Article ID 904320 (2011)

9. Gürkan, G, Özge, AY, Robinson, SM: Sample path solution of stochastic variational inequalities, with applications to option pricing. In: Charnes, JM, Morice, DM, Brunner, DT, Swai, JJ (eds.) Proceedings of 1996 Winter Simulation Conference, pp. 337-344 (1996)

10. Robinson, SM: Analysis of sample-path optimization. In: Ruszezynski, A, Shaprio, A (eds.) Handbooks in Operations Research and Management Science: Stochastic Programming, pp. 353-425. Elsevier, New York (2003)

11. Shaprio, A: Monte Carlo sampling methods. J. Math. Anal. Appl. 167, 582-587 (1992)

12. Jiang, $\mathrm{H}, \mathrm{Xu}, \mathrm{H}$ : Stochastic approximation approaches to the stochastic variational inequality problems. IEEE Trans. Autom. Control 53, 1462-1475 (2008)

13. $\mathrm{Xu}, \mathrm{H}$ : Sample average approximation methods for a class of stochastic variational inequality problems. Asia-Pac. J. Oper. Res. 27, 103-119 (2010)

14. Shaprio, A, Xu, H: Stochastic mathematical programs with equilibrium constraints, modeling and sample average approximation. Optimization 57, 395-418 (2008)

15. Chen, S, Pang, L, Guo, F, Xia, Z: Stochastic methods based on Newton method to the stochastic variational inequality problem with constraint conditions. Math. Comput. Model. 55, 779-784 (2012)

16. Chen, F, Yan, H, Yao, L: A newsvendor pricing game. IEEE Trans. Syst. Man Cybern., Part A, Syst. Hum. 34, 1181-1191 (2004)

17. Mahajan, S, Van Ryzin, G: Inventory competition under dynamic consumer choice. Oper. Res. 49, 464-657 (2001)

18. Dong, J, Zhang, D, Nagurney, A: A supply chain network equilibrium model with random demands. Eur. J. Oper. Res. $156,194-212(2004)$

19. Walting, D: User equilibrium traffic network assignment with stochastic travel times and late arrival penalty. Eur. J. Oper. Res. 175, 1539-1556 (2006)

20. Ngo, MH, Krishnamarthy, VK: Game theoretical cross-layer transmission policies in multi-reception wireless networks. IEEE Trans. Signal Process. 55, 1911-1926 (2007)

21. Liu, B: Uncertainty Theory, 2nd edn. Springer, Berlin (2007)

22. Fukushima, M: Equivalent differentiable optimization problems and descend methods for asymmetric variational inequality problems. Math. Program. 53, 99-110 (1992)

23. Liu, B: Uncertainty Theory: A Branch of Mathematics for Modeling Human Uncertainty. Springer, Berlin (2010)

24. Liu, B: Some research problem in uncertainty theory. J. Uncertain Syst. 3(1), 3-10 (2009)

25. Liu, Y, Ha, M: Expected value of function of uncertain variables. J. Uncertain Syst. 4(3), 181-186 (2010)

26. Fukushima, M: Fundamentals of Nonlinear Optimization. Asakura Shoten, Tokyo (2001) (in Japanese); Chinese edition: translated by GH Lin, published by Science Press, Beijing (2011)

\section{Submit your manuscript to a SpringerOpen ${ }^{\circ}$ journal and benefit from:}

- Convenient online submission

Rigorous peer review

- Immediate publication on acceptance

- Open access: articles freely available online

- High visibility within the field

- Retaining the copyright to your article 